\title{
Parâmetros e Tendência Genética da Produção de Leite de Cabra no Brasil ${ }^{1}$
}

\section{Heraldo Cesar Gonçalves ${ }^{2}$, Martinho de Almeida e Silva ${ }^{3}$, Francisco Stefano Wechsler ${ }^{2}$, Alcides Amorim Ramos ${ }^{2}$, Lilian Martini Pulz ${ }^{4}$, Tiago Creste Losi ${ }^{4}$}

RESUMO - A caprinocultura leiteira no Brasil, apesar de ser uma atividade rural consolidada há algumas décadas, tem se mostrado totalmente dependente de outros países no que se refere ao melhoramento genético. A maioria dos plantéis existentes atualmente tem como base animais importados, e a renovação do material genético é feita por meio da importação de sêmen. Inexistem informações sobre o valor genético dos animais e sua evolução no decorrer dos anos. No presente trabalho, foram estimadas a herdabilidade e a repetibilidade da produção de leite utilizando o REML. Os valores obtidos foram 0,21557 e 0,21564, respectivamente. Para a predição do valor gênico dos animais, foi usado o procedimento BLUP com modelo animal. A mudança na tendência genética anual estimada por um modelo quadrático foi $-0,8109 \mathrm{~kg} / \mathrm{ano}^{2}$, indicando desaceleração no ganho genético. A correlação de Pearson entre os valores gênicos dos bodes estimados com base na média da capacidade provável de produção das filhas obtida pelo método de mínimos quadrados com as estimadas pelas equações do modelo misto foi de 0,5751 . A correlação de SPEARMAN entre as classificações dos bodes obtidos pelos dois métodos foi de 0,5813 .

Palavras-chave: herdabilidade, repetibilidade, valor gênico

\section{Genetic Parameters and Trend for Goat Milk Production in Brazil}

\begin{abstract}
Dairy goat production in Brazil, although firmly established since a few decades, has proved to be totally dependent on foreign countries for genetic improvement. Most herds presently producing are based on imported animals, and genetic refreshment is obtained through imported semen. No information is available on the genetic values and their evaluation along the years. In this paper, heritability and repeatability estimates, obtained through REML, are reported for milk yield. The values were 0.21557 and 0.21564 , respectively. The BLUP method with animal model was used to predict genetic values. The annual change in genetic trend, estimated by means of a quadratic model, was $-0.8109 \mathrm{~kg} / \mathrm{year}^{2}$, which indicates a slowdown in genetic gain. The Pearson correlation between buck genetic values estimated by means of the most likely production of daughters and least squares and those estimated through BLUP and mixed model equations was 0.5751 ; the Spearman correlation between buck ranks obtained by both methods was 0.5813 .
\end{abstract}

Key Words: heritability, repeatability, genetic values

\section{Introdução}

A caprinocultura leiteira no Brasil, nas últimas três décadas, vem se consolidando como atividade rentável, despertando o interesse de muitos produtores rurais. Esta atividade está alicerçada na exploração de raças caprinas exóticas, especializadas na produção de leite e a melhoria dos plantéis, com base na importação de animais e sêmen, principalmente da França, onde existe um esquema nacional de avaliação de reprodutores.

Dessa forma, a melhoria dos plantéis brasileiros é completamente dependente de outros países, cujos objetivos da seleção nem sempre interessam aos produtores nacionais (Gonçalves, 1996).
São escassas as estimativas de parâmetros genéticos na literatura nacional e muito pouco se conhece sobre evolução genética dos animais no Brasil.

Os valores de herdabilidade situam-se entre 0,16 e 0,31 (Boichard et al., 1989; Bishop et al., 1994; Moioli et al., 1995; Analla et al., 1996) e os de repetibilidade, entre 0,28 e 0,65 (Bishop et al., 1994; Moioli et al., 1995; Analla et al., 1996 ).

Wiggans et al. (1984), nos Estados Unidos, avaliaram bodes das raças Parda Alpina, La Mancha, Anglo Nubiana, Saanen e Toggenburg, por meio do procedimento BLUP, usando herdabilidade de $0,20 \mathrm{e}$ repetibilidade de 0,50 . $\mathrm{Na}$ análise realizada, considerando ou não o parentesco entre os bodes, esses autores encontraram, para a produção de leite, corre-

\footnotetext{
1 Parte da tese apresentada à UFV pelo primeiro autor, para obtenção do título de Doctor Scientiae". Trabalho financiado pela FAPEMIG. 2 Professor da FMVZ - UNESP - Campus de Botucatu, 18618-000 - Botucatu, SP. E.mail: heraldo@fca.unesp.br

3 Professor da UFMG - Belo Horizonte, MG. E.mail: martinho@vet.ufmg.br

${ }^{4}$ Discente de Zootecnia da FMVZ - UNESP - Campus de Botucatu, 18618-000 - Botucatu, SP
} 
lação média de 0,86 entre os valores gênicos para todas as raças analisadas. Segundo esses autores, com base nessa correlação, a inclusão ou não do parentesco influi na solução das equações de modelos mistos.

A tendência genética para produção de leite relatada por Wiggans et al. (1988) foi de 1,$17 ;-1,18$; 0,20; 4,02; e 2,01 kg/ano para as raças Parda Alpina, La Mancha, Anglo Nubiana, Saanen e Toggenburg, respectivamente, nos Estados Unidos.

Outra avaliação da tendência genética realizada por Wiggans (1989), dessa vez ajustando a média dos valores genéticos por ano de forma quadrática e incluindo avaliação de animais mestiços, apontou os valores de 0,$48 ; 0,89 ; 0,16 ; 0,67 ;-0,73 ; 0,15 ; \mathrm{e} 0,42 \mathrm{~kg} /$ ano $^{2}$ para as raças Parda Alpina, Experimental, La Mancha, Anglo Nubiana, Oberhasli, Saanen e Toggenburg, respectivamente.

$\mathrm{Na}$ Itália, trabalhando com animais da raça Saanen, Moioli et al. (1995) obtiveram o valor de 0,7 1/ano, calculado por meio de regressão linear do valor genético médio por ano de nascimento.

Assim, os objetivos deste trabalho foram estimar a herdabilidade e repetibilidade da produção de leite por meio de componentes de variância obtidos pelo método da máxima verossimilhança restrita (REML); classificar geneticamente os bodes usados como reprodutores no Brasil, por dois métodos, estimando a correlação entre eles; e verificar a tendência genética da produção de leite.

\section{Material e Métodos}

Os dados foram coletados em sete propriedades localizadas na região Sudeste do Brasil.

Em todas as propriedades, os animais foram criados no sistema confinado em aprisco suspenso do solo, com piso ripado, ordenhados duas vezes ao dia, com os cabritos aleitados artificialmente com leite natural ou artificial.

Embora existissem algumas diferenças entre as fazendas participantes deste estudo, a alimentação fornecida era basicamente a mesma, atendendo as necessidades nutricionais dos animais nas diversas categorias. Até o $10^{\circ}$ dia de vida, os cabritos receberam apenas leite. A partir do $11^{\circ}$ dia, foram colocados feno e concentrado à vontade. A desmama foi realizada entre 60 e 90 dias, desde que os animais apresentassem pelo menos 2,5 vezes o peso ao nascer.

Após o desmame, as fêmeas receberam feno de gramínea ou leguminosa à vontade e por volta de 400 g/dia de concentrado com $18 \%$ de proteína bruta. Quando as cabritas atingiram peso entre 30 e $35 \mathrm{~kg}$, iniciava-se sua vida reprodutiva. Durante a gestação, a cabrita continuava a receber a mesma alimentação até a parição e, após o parto, a alimentação volumosa era constituída de capim-elefante e feno de gramíneas e leguminosas. $\mathrm{O}$ alimento concentrado, fornecido na hora da ordenha, foi suficiente para complementar as necessidades nutricionais dos animais. A mistura mineral fazia parte da suplementação alimentar de todas as categorias.

Em todas as propriedades, os animais receberam tratamento contra parasitas intestinais e pulmonares.

Foram analisados dados da produção de leite, oriundos de fichas zootécnicas mantidas pelos criadores e do controle oficial realizado pela Associação Brasileira de Criadores. Inicialmente, os dados foram ajustados, individualmente, de acordo com a função difásica (Grossman \& Koops, 1988), com o intuito de estimar a produção de leite total da lactação.

Somente os reprodutores com pelo menos duas filhas participaram da análise. Assim, trabalhou-se com 1.336 lactações, de 678 cabras, filhas de 95 reprodutores, em sete fazendas, durante sete anos (de 1986 a 1992) e pertencentes a três raças ( 1 = Parda, 2 = Saanen e 3 = Toggenburg).

Para as estimativas dos parâmetros da função difásica, foi usado o procedimento REGREGN do programa SAEG - Sistema para Análise Estatística e Genética (UFV, 1997), que utiliza o método de Gauss-Newton puro.

De posse dos parâmetros da função difásica, calculou-se a produção de leite total por meio da integral da equação (1):

$\operatorname{PLT}_{\mathrm{DL}}=\sum_{\mathrm{i}=1}^{2}\left\{\mathrm{a}_{\mathrm{i}}\left[\tanh \left(\mathrm{b}_{\mathrm{i}}\left(\mathrm{DL}-\mathrm{c}_{\mathrm{i}}\right)\right)-\tanh \left(\mathrm{b}_{\mathrm{i}}\left(0-\mathrm{c}_{\mathrm{i}}\right)\right)\right]\right\}$,

em que DL = duração da lactação; tanh = tangente hiperbólica; para cada fase i; $a_{i}, b_{i}$ e c $c_{i}=$ parâmetros a serem estimados pela regressão não-linear, em que $\mathrm{i}=1$ e 2 .

Os valores gênicos dos reprodutores foram obtidos por dois métodos: 1) método da Capacidade Provável de Produção (CPP) das filhas e 2) BLUP, por meio da solução das equações de modelo misto para o modelo de repetibilidade (Quass, 1984) na forma univariada.

As estimativas dos componentes de variância

R. Bras. Zootec., v.31, n.6, p.2204-2208, 2002 
para o cálculo da repetibilidade usada no método (1) foram obtidas pelo método Henderson III, utilizando o programa LSMLMN (Harvey, 1990). Para esse método, as produções das filhas foram corrigidas para os efeitos fixos e calculou-se CPP para cada filha, com base no valor da repetibilidade.

A fórmula usada para o cálculo das CPP das filhas foi:

$$
C P P=u+b_{\hat{Y} i} \bar{Y}_{i} .\left(\bar{Y}_{i}-u\right)
$$

em que $\mathrm{u}=$ constante inerente a todas as observa-

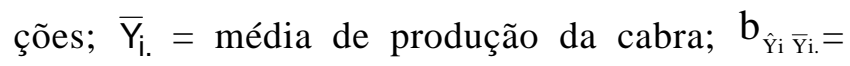
coeficiente de regressão da produção de uma lactação futura, em função da média de produções da cabra, sendo:

$$
\mathrm{b}_{\hat{\mathrm{Y}}_{\mathrm{i}} \overline{\mathrm{Y}}_{\mathrm{i} .}}=\frac{\mathrm{n} \hat{\mathrm{r}}}{1+(\mathrm{n}-1) \hat{\mathrm{r}}}
$$

em que $\mathrm{n}=$ número de observações da cabra; e $\hat{\mathrm{r}}=$ repetibilidade da característica.

O valor genético dos bodes foi obtido pela fórmula:

$$
V G=u+b_{A i} \bar{F}\left(\bar{P}_{F}-u\right)
$$

em que $\mathrm{u}=$ constante inerente a todas as observações; $\bar{P}_{F}=$ média da $\mathrm{CPP}$ das filhas; $b_{\text {Ai } \bar{F}}=$ regressão do valor genético do bode, em função da CPP de suas filhas. Considerando que esse valor não é correlacionado com o ambiente permanente de suas filhas, tem-se:

$$
\mathrm{b}_{\mathrm{A}_{\mathrm{i}} \overline{\mathrm{F}}}=\frac{2 n \hat{\mathrm{h}}^{2}}{4 \hat{\mathrm{r}}+(\mathrm{n}-1) \hat{\mathrm{h}}^{2}}
$$

em que $\mathrm{n}=$ número de filhas do bode; $\hat{\mathrm{h}}^{2}=$ herdabilidade da característica; $\mathrm{e} \hat{\mathrm{r}}=$ repetibilidade da característica.

Para o método (2), as estimativas de componentes de variância e covariância foram obtidas pelo programa Multiple Trait Derivative-Free Restricted Maximum Likelihood (MTDFREML), desenvolvido por Boldman et al. (1993), que adotam o procedimento livre de derivada (DF). O programa usa o modelo animal e pode ser empregado para modelos uni ou multivariados, com ou sem medidas repetidas por animal, permitindo também obter a solução dos efeitos fixos e a predição dos valores genéticos dos animais. Os componentes de variância estimados pelo método dos quadrados mínimos (Henderson III) foram usados como valores iniciais para este procedimento.

O critério de convergência adotado foi de $10^{-6}$ e, após o estabelecimento deste, para evitar a ocorrência de máximo local, foram realizados novos processamentos, tendo sido encerrado o processo, quando os resultados dos dois últimos processamentos eram iguais.

O cálculo dos componentes de variância e covariância, estimação dos efeitos fixos e predição do valor genético dos animais para a produção de leite total, foi realizado empregando-se o modelo matricial (1):

$$
y=X \beta+Z g+W p+e
$$

em que $\mathrm{y}=$ vetor de observações de características medidas nos indivíduos; $\mathrm{X}=$ matriz de incidência dos efeitos fixos conhecida; $\beta$ = vetor de efeitos fixos desconhecidos: ano, estação, fazenda, raça e idade como covariavel; $Z=$ matriz de incidência dos valores genéticos aleatórios conhecida; $g$ = vetor de valores genéticos aditivos desconhecidos; $\mathrm{W}=$ matriz de incidência dos efeitos de ambiente permanente conhecida, sendo $Z=[0 \mathrm{~W}] ; \mathrm{p}=$ vetor de efeito de ambiente permanente desconhecido; e e = vetor de ambiente temporário desconhecido.

Assume-se que:

$$
\begin{aligned}
& \mathrm{E}(\mathrm{y})=\mathrm{X} \\
& \mathrm{E}(\mathrm{g})=\mathrm{E}(\mathrm{p})=\mathrm{E}(\mathrm{e})=0 \\
& \mathrm{~V}(\mathrm{~g})=\mathrm{A} \sigma_{\mathrm{g}}^{2} \\
& \mathrm{~V}(\mathrm{p})=\boldsymbol{\sigma}_{p}^{2} \\
& \mathrm{~V}(\mathrm{e})=\mathrm{I} \sigma_{\mathrm{e}}^{2}
\end{aligned}
$$

em que $\mathrm{A}=$ matriz que indica o grau de parentesco entre os indivíduos de ordem $\mathrm{s} ; \mathrm{Z}=$ matriz de incidência dos valores genéticos de ordem $\mathrm{n}$ x s; $\mathrm{W}=$ matriz de incidência dos efeitos permanentes de ambiente de ordem $\mathrm{n} \times \mathrm{p} ; \mathrm{I}=$ matriz-identidade de ordem $\mathrm{n} ; \mathrm{I}_{\mathrm{p}}=$ matriz-identidade de ordem $\mathrm{p} ; \sigma_{\mathrm{g}}^{2}=$ variância genética aditiva; $\sigma_{p}^{2}=$ variância de ambiente permanente; e $\sigma_{\mathrm{e}}^{2}=$ variância de ambiente temporário; em que $\mathrm{n}=$ número total de observações; $\mathrm{p}=$ número de indivíduos com observações; e s = número total de indivíduos da população (com ou sem informação). 
Para o cálculo da correlação do valor gênico dos bodes, como indicado acima, com as obtidas pelo BLUP, foram empregados os métodos de PEARSON (paramétrica) e de SPEARMAN (não paramétrica). Empregou-se o programa SAEG - Sistema para Análise Estatística e Genética (UFV, 1997) para a obtenção dos coeficientes de correlação.

A tendência genética foi calculada por meio de regressão quadrática do valor genético médio das cabras sobre o ano de nascimento. A declividade (tendência) para um ano específico foi calculada pela derivada primeira e a mudança anual, pela derivada segunda da equação de regressão (Wiggans, 1989).

Estimação dos componentes de variância $e$ covariância e parâmetros genéticos

As estimativas dos componentes de variância, herdabilidade e repetibilidade estimadas para a produção de leite foram: $\hat{\sigma}_{g}^{2}=16887,6873, \hat{\sigma}_{p}^{2}=5,3630$, $\hat{\sigma}_{e}^{2}=61443,0149, \hat{h}^{2}=0,21557, \hat{r}=0,21564$.

A estimativa de herdabilidade da produção de leite obtida pelo REML foi menor que a obtida pelo método de Henderson III (Gonçalves et al., 2001) para o mesmo conjunto de dados.

Esse resultado é compreensível, visto que, no método Henderson III, as produções repetidas de um mesmo indivíduo são tomadas como se fossem produções de meias-irmãs, fazendo com que haja incremento no componente de variância de reprodutor, enquanto no modelo animal se tomam corretamente as produ-

Tabela 1 - Número de animais e tendência genética da produção de leite total por ano de nascimento das cabras

Table 1 - Number of animals and genetic trend of milk production, according to the goats birth year

\begin{tabular}{lcc}
\hline $\begin{array}{l}\text { Ano de nascimento } \\
\text { Birth year }\end{array}$ & $\begin{array}{c}\text { Número de } \\
\text { animais } \\
\text { Number of animals }\end{array}$ & $\begin{array}{c}\text { Tendência } \\
\text { genética (kg/ano) } \\
\text { Genetic trend } \\
(\text { kg/year })\end{array}$ \\
\hline 83 & 34 & 6,510 \\
84 & 49 & 5,690 \\
85 & 81 & 4,880 \\
86 & 125 & 4,070 \\
87 & 74 & 3,260 \\
88 & 94 & 2,450 \\
89 & 83 & 1,640 \\
90 & 36 & 0,830 \\
91 & 35 & 0,018 \\
\hline
\end{tabular}

R. Bras. Zootec., v.31, n.6, p.2204-2208, 2002 ções repetidas como sendo de um mesmo indivíduo.

Com relação à estimativa de repetibilidade obtida pelo REML (Gonçalves et al., 2001), embora tenha sido realizada em um arquivo de dados diferente do método Henderson III, ela também mostrou-se menor.

Com os baixos valores obtidos para a repetibilidade, constata-se a baixa confiabilidade em poucas observações dessa característica. Por outro lado, em termos práticos, o aumento do número de observações por animal acarretaria aumento do intervalo de gerações e diminuição do ganho genético anual.

Correlação entre a classificação dos bodes pelo valor gênico obtido com base na média da CPP de suas filhas com a classificação obtida pelo BLUP

A correlação de PEARSON entre os valores genéticos analisados foi de 0,5751 e a correlação de SPEARMAN entre classificações, de 0,5813 , ambas significativas $(\mathrm{p}<0,01)$.

Embora significativos, os baixos valores estimados deduzem que os métodos classificaram de forma bastante diferente os candidatos à seleção, possivelmente em virtude de o método das CPP não levar em consideração o parentesco dos ancestrais e colaterais.

Tendência genética da produção de leite total

$\mathrm{Na}$ Tabela 1 são apresentados os valores da tendência genética (primeira derivada) ajustados por uma curva quadrática, cujo gráfico se encontra na Figura 1. A mudança anual na tendência genética (segunda derivada) foi de $-0,8109 \mathrm{~kg} / \mathrm{ano}^{2}$, indicando

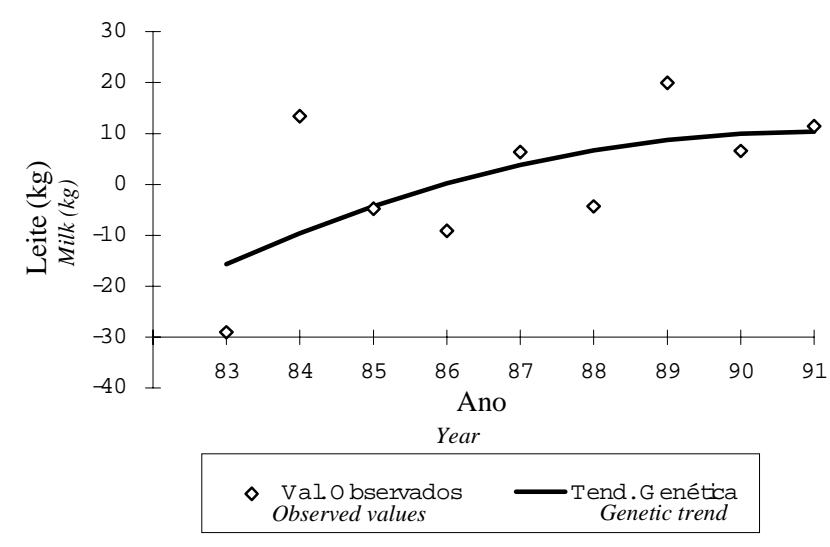

Figura 1 - Tendência genética da produção de leite total por ano de nascimento das cabras.

Figure 1 - Genetic trend of total milk production according to the goats birth year. 
desaceleração no ganho genético. Valor semelhante em magnitude e com mesmo sinal foi relatado por Wiggans (1989) em relação à raça Oberhasli.

Essa redução no ganho genético anual possivelmente reflete a ausência de um programa eficaz de seleção dos animais no Brasil, que, até o presente, se caracterizou pela importação de reprodutores e sêmen de outros países. Sabe-se que a maior parte do material genético importado pelo Brasil provém da França, onde a seleção visa principalmente melhorar a taxa dos constituintes do leite, especialmente o teor protéico, sendo que a produção de leite está em segundo plano. Talvez essa seja a principal razão do declínio no ganho genético anual.

\section{Conclusões}

Considerando o valor da herdabilidade da produção de leite de baixo a moderado, e por esta característica limitar-se ao sexo, sugere-se o teste de progênie como método de seleção mais eficiente para a seleção dos bodes e o uso da inseminação artificial como método de disseminação do material genético selecionado.

A tendência genética negativa reduziu o ganho anual, indicando ausência de um programa eficaz de seleção dos animais no Brasil. Seria aconselhável modificar o método de seleção atualmente adotado, visando à reversão dessa situação.

Os valores gênicos dos reprodutores obtidos pelos dois métodos (Capacidade Provável de Produção das Filhas e BLUP) apresentaram diferenças na classificação dos reprodutores.

\section{Literatura Citada}

ANALLA, M.; JIMÉNEZ-GAMERO, I.; MUÑOS-SERRANO, A. et al. Estimation of genetic parameters for milk yield and fat and protein contents of milk from Murciano-Granadina goats. Journal of Dairy Science, v.79, p.1895-1898, 1996.

BISHOP, S.; SULLIVAN, B.P.; SCHAEFFER, L.R. et al. Genetic evaluation of Canadian dairy goats using test-day data. In: INTERNATIONAL COMMITTEE FOR ANIMAL RECORDING, 1994, Ottawa. Proceedings...Ottawa: Agriculture and Agri-Food Canada, Dairy Division, 1994. p.299-302.

BOICHARD, D.; BOULOC, N.; RICORDEAU, G. et al. Genetic parameters for first lactation dairy traits in the Alpine and Saanen goat breeds. Génétique, Sélection, Évolution, v.21, p. 205-215, 1989.
BOLDMAN, K.G.; KRIESE, L.A.; Van VLECK, L.D. et al. A Manual for use of MTDFREML; a set of programs to obtain estimates of variances and covariances. Lincoln: Department of Agriculture, Agricultural Research Service, 1993. p. 120.

GONÇALVES, H.C. Fatores genéticos e de meio em algumas características produtivas e reprodutivas de caprinos. Viçosa, MG: Universidade Federal de Viçosa, 1996. 141p. Tese (Doutorado em Zootecnia) - Universidade Federal de Viçosa, 1996.

GONÇALVES, H.C.; SILVA, M.A.; WECHSLER, F.S. et al. Fatores genéticos e de meio na produção de leite de caprinos leiteiros. Revista Brasileira de Zootecnia, v.30, n.3, p.719-729, 2001.

GROSSMAN, M.; KOOPS, W.J. Multiphasic analysis of lactation curves in dairy cattle. Journal of Dairy Science, v.71, p.1598-1608, 1988.

HARVEY, W. R. User's guide for LSMLMW and MIXMDL PC - 2 version. Ohio: State University, 1990. 91p.

MOIOLI, B.M.; PILLA, A.M.; ROSATI, A. et al. Ereditabilità e ripetibilità della produzione di latte nella razza caprina Saanen e valutazione genetica dei riproduttori. Zootecnica e Nutrizione Animale, v.21, p.231-236, 1995.

QUAAS, R.L. Linear prediction. In: QUAAS, R.L.; ANDERSON, R.D.; GILMOUR, A.R. (Eds.) Use of mixed models for prediction and for estimation of (co)variance components. s.1., University of New England, AGBU, 1984. p.1-76. (BLUP School Handbook).

UNIVERSIDADE FEDERAL DE VIÇOSA - UFV. SAEG Sistema de análises estatísticas e genéticas. Versão 7.1. Viçosa, MG: 1997. 150p. (Manual do usuário).

WIGGANS, G.R. Animal model evaluation of dairy goats for milk, fat, and protein yields with crossbred animals included. Journal of Dairy Science, v.72, p.2411-2416, 1989.

WIGGANS, G.R.; DICKINSON, F.N.; KING, J. et al. Genetic evaluation of dairy goat bucks for daughter milk and fat. Journal of Dairy Science, v.21, p.201-207, 1984.

WIGGANS, G.R.; VAN DIJK, J.W.J.; MISZTAL, I. Genetic evaluation of dairy goats for milk and fat yield with an animal model. Journal of Dairy Science, v.71, p.1330-1337, 1988.

Recebido em: 04/09/01

Aceito em: 09/09/02 